PRAXIS

ecucativa

Universidad Nacional de La Pampa

Facultad de Ciencias Humanas

Instituto de Ciencias de la Educación

para la investigación interdisciplinaria

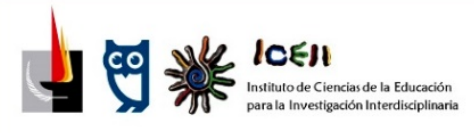

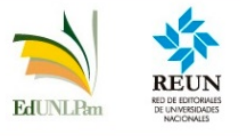

ISSN 2313-934X

SANTA ROSA, LA PAMPA, ARGENTINA

Correo electrónico: iceii@humanas.unlpam.edu.ar

Disponible en https://cerac.unlpam.edu.ar/index.php/praxis

Pedagogías del conflicto ambiental: aportes desde una experiencia participativa de formación docente en un territorio en disputa. Artículo de Luciano Iribarren, Katherine Guerrero Tamayo, Fernando Garelli y Ana Dumrauf. Praxis educativa, Vol. 26, No 1 enero - abril 2022. E - ISSN 2313-934X. pp. 1-24. https://dx.doi.org/10.19137/praxiseducativa-2022-260102

\title{
Pedagogías del conflicto ambiental: aportes desde una experiencia participativa de formación docente en un territorio en disputa
}

Pedagogies of the environmental conflict: contributions from a participatory experience of teacher training in a disputed territory

Pedagogias do conflito ambiental: contribuições de uma experiência participativa da formação de professores em um território disputado

\section{Luciano Iribarren}

Universidad Nacional de La Plata, Consejo Nacional de Ciencia y Tecnología, CONICET, Argentina

luciano.iribarren@gmail.com

ORCID 0000-0003-4244-1325

\section{Katherine Guerrero Tamayo}

Universidad Nacional de La Plata, Consejo Nacional de Ciencia y Tecnología, CONICET, Argentina

kathyagueta@gmail.com

ORCID: 0000-0001-9259-8895

\section{Fernando Garelli}

Universidad Nacional de La Plata, Consejo Nacional de Ciencia y Tecnología, CONICET, Argentina

fgarelli@gmail.com

ORCID 0000-0002-7332-2073 


\section{Ana Dumrauf}

Universidad Nacional de La Plata, Consejo Nacional de Ciencia y Tecnología, CONICET, Argentina

adumrauf@iflysib.unlp.edu.ar

ORCID 0000-0002-4856-787X

Recibido: 2021-07-13 | Revisado: 2021-08-09 | Aceptado: 2021-09-07

\section{Resumen}

Presentamos una primera sistematización de un proceso de formación docente en ejercicio, surgido de la articulación entre una organización socioterritorial y una escuela en el Delta del Paraná. Este territorio de extensos humedales se encuentra atravesado por disputas entre espacios de vida campesina, portadora de modos de vida isleños, y avanzadas de privatización hacia barrios cerrados de lujo con graves consecuencias socioambientales. Pretendemos aportar a la comprensión algunas maneras en las que estos movimientos pueden contribuir a los marcos pedagógico-didácticos que sustentan diálogos entre escuela y comunidades. En particular, analizamos los aportes a la pedagogización de los conflictos ambientales.

Palabras clave: educación ambiental, conflictos ambientales, pedagogías del conflicto ambiental, formación docente, educación comunitaria.

\section{Abstract}

We present a first systematization of an in-service teacher training process, arising from the articulation between a socio-territorial organization and a school in the Paraná Delta. This territory of extensive wetlands is crisscrossed by disputes between on one side rural living spaces and islander ways of life and on the other side privatization and transformation into luxury gated communities with serious socio-environmental consequences. We intend to contribute to the understanding of some ways in which these socio-territorial movements can contribute to the pedagogical-didactic frameworks that sustain dialogues between school and communities. In particular, we analyze the contributions to the pedagogization of environmental conflicts.

Keywords: environmental education, environmental conflicts, pedagogies of the environmental conflict, teacher training, community education.

\section{Resumo}

Apresentamos uma primeira sistematização de um processo de formação de professores em serviço, decorrente da articulação entre uma organização sócio-territorial e uma escola no Delta do Paraná. Esse território de extensas áreas úmidas é atravessado por disputas entre os espaços de vida camponeses com estilos de vida insulares e a privatização para bairros fechados de luxo com sérias conseqüências socioambientais. Pretendemos contribuir para a compreensão de algumas maneiras pelas quais esses movimentos podem contribuir para as estruturas pedagógico-didáticas que sustentam o diálogo entre a escola e as comunidades. Em particular, analisamos as contribuições para a pedagogização de conflitos ambientais.

Palavras-chave: educação ambiental, conflitos eco-territoriais, pedagogias do conflito ambiental, formação de professores, educação comunitária. 


\section{Introducción}

Desde hace algunos años, como Grupo de Didáctica de las Ciencias (GDC), participamos de procesos de construcción de propuestas político-pedagógicas para la Educación en Ciencias Naturales, Ambiental y en Salud (ECNAS) que pretenden sostener una intencionalidad emancipadora. Entendemos dicha intencionalidad como "un movimiento colectivo e individual, de liberación consciente y de superación de las formas de alienación material y simbólica" (Loureiro, 2003, p. 22). En este camino, comenzamos a considerar que los enfoques para la educación científica en Latinoamérica necesitan privilegiar el análisis crítico e integral de las crisis y conflictos ecoterritoriales regionales, a fin de comprender realidades próximas, propias y de manera compleja, vistas como parte de la situación de emergencia planetaria (Dumrauf y Mengascini, 2008).

Desde mediados de los 70, la globalización neoliberal produjo grandes transformaciones de los espacios de vida a escala global, reestructurando los soportes materiales y simbólicos en un nuevo ciclo de redefinición del colonialismo. La biodiversidad y riqueza ecológica latinoamericana adquirió un alto valor estratégico "como espacio subalterno de aprovisionamiento y subsidio de bienes y servicios ambientales capaces de 'alimentar' un nuevo ciclo de expansión capitalista" (Machado Aráoz, 2013, p. 31). Frente a ello, se erigieron diversos movimientos sociales y populares en toda la región. Se trata de grupos heterogéneos en cuanto a orígenes, bases ideológicas, condiciones de clase, maneras de organizarse, acciones y estrategias políticas. La movilización en rechazo a las formas más agresivas del extractivismo (megaproyectos mineros, petroleros, hidroeléctricas, agronegocios, etc.) ha sido su primer punto de convergencia, y el escenario para su constitución y organización.

Estos movimientos han implementado numerosas propuestas educativas que nos llevan a pensar transformaciones en la educación (Rockwell, 2012). Las investigaciones realizadas por Guelman y Palumbo (2015) afirman que, como parte de su intencionalidad transformadora, lo pedagógico evidencia su trama política al cruzar el accionar de los movimientos en la construcción permanente de una nueva cultura, de una intención política contrahegemónica y prefigurativa que pone en acto el papel formador de la cotidianeidad.

En nuestro recorrido, hemos sostenido procesos de investigación con instancias de participación en el campo de la ECNAS, con movimientos sociales (Dumrauf et al., 2016; Garelli et al., 2019) y con docentes en ejercicio (Dumrauf et al., 2013; Dumrauf y Cordero, 2018; Dumrauf y Cordero, 2020). Sin embargo, hasta el momento, la articulación de ambas líneas de trabajo, más allá de marcos teórico-metodológicos compartidos, no había sido posible. ¿Cómo articular organizaciones sociales en lucha con la escuela? ¿Cómo con instancias de formación docente en ejercicio? ¿Qué aportaría, si lo hiciera, este proceso de trabajo conjunto a los marcos pedagógicodidácticos que sustentan estos diálogos entre escuela y comunidad? Estas preguntas orientaron las reflexiones que, pretendemos, compartirmos en este artículo. Nos basamos, en un primer análisis, de un proceso de formación docente continua en Educación Ambiental que realizamos en 
una escuela pública de gestión estatal del Delta del Río Paraná (Islas de Tigre, Buenos Aires) junto con, y a instancias de, una organización socioterritorial.

A continuación, enunciamos algunos antecedentes y fundamentos sobre Educación Ambiental desde perspectivas críticas que sustentaron nuestras prácticas; luego, proporcionaremos una breve historización del conflicto ecoterritorial en el marco del cual se configuró el proceso de formación que analizamos; presentamos algunas notas acerca de la metodología implementada; mostramos la forma en que fue construido y las características principales del dispositivo de formación; identificamos los emergentes surgidos en la implementación respecto de categorías de análisis provenientes de la Pedagogía del Conflicto Ambiental (Canciani y Telias, 2013; Canciani et al., 2017); y, finalmente, presentamos una breve discusión acerca de los aportes de este análisis a la construcción de pedagogías del conflicto ambiental.

\section{Educación Ambiental desde perspectivas críticas: algunos antecedentes y fundamentos}

Nuestro interés más general en la socialización del análisis de nuestras experiencias es el de aportar a una Educación Ambiental (EA) entendida como práctica política, epistemológica y ontológica que intenta construir una racionalidad ambiental (Leff, 2014) que promueva el cuidado de la vida y la descolonización de los territorios del Sur. Esta EA pretende romper la experiencia del saber centralizado, constituido en saber-poder de control y regulación de lo ambiental para recomponer miradas que hablan de otros mundos posibles (Escobar, 2018). Dichas miradas consideran otras formas de habitar que se entienden como disidentes de las voces oficiales. Integran, en la escuela (y la universidad), modos de comprensión de la cuestión ambiental que emergen desde las organizaciones sociales-culturales. Según Leff (2018), la racionalidad ambiental territorializa la ontología de la diversidad, la política de la diferencia y la ética de la otredad en la forja de nuevos mundos de vida. Estos posicionamientos, amalgamados en lo que se conoce como Pensamiento Ambiental Latinoamericano (PAL), toman distancia del discurso hegemónico del desarrollo sostenible, apartándose de la mirada unidisciplinar para dar paso a la transdisciplinariedad colaborativa y al diálogo de saberes (Leff, 2014, 2017, 2018). El diálogo de saberes diversos donde se encuentren los saberes expertos con los saberes populares, campesinos e indígenas acerca de lo ambiental sería, para este autor, una vía para la construcción de saber ambiental.

Por ello, sustentamos la EA enmarcada en el Pensamiento Ambiental Latinoamericano, en los marcos político-pedagógicos de la educación popular de perspectiva freireana, con la que comparte el horizonte transformador de la sociedad que impulsan los movimientos socioterritoriales. Paulo Freire, desde sus primeros textos a sus últimas "cartas pedagógicas", nos recordó que no alcanza con la educación para transformar al mundo, pero sin ella sería imposible (Freire, 1968, 2000). Por su horizonte ético-político, no es de sorprender que podamos rastrear los orígenes de la educación popular latinoamericana en la larga historia de liberación de América (Puiggrós, 2005). Esta perspectiva cuenta ya con un acumulado de teorías, conceptos, 
metodologías y experiencias pedagógicas llevadas adelante por más de medio siglo (Mejía, 2014). Una de las bases de este acumulado es que la educación solo será transformadora y permitirá construir un mundo más justo, menos desigual y más respetuoso de las diferencias si se construye a través de la participación, la mirada y los intereses de los grupos que sufren las opresiones y ven vulnerados sus derechos, sus vidas, sus cuerpos.

Sin embargo, Corbetta (2015) reconoce grandes dificultades para la implementación de una EA con anclaje territorial, situada y pensada desde el Sur que: reconozca saberes otros; sea capaz de abordar críticamente el modo de ser, estar y habitar los territorios; se posicione frente a la visión de mundo eurocéntrica y reconozca otras visiones; aborde pedagógicamente los conflictos ecoterritoriales que se viven en la región; e identifique actores, significados en pugna en torno a la naturaleza y dilemas éticos que conlleva el "desarrollo". De acuerdo con la autora, resulta dificultoso abrir, en las escuelas, debates acerca de los esfuerzos necesarios para el diálogo interdisciplinar, interinstitucional y de saberes diversos que la EA, desde la perspectiva PAL, demanda.

Abordando este desafío, Canciani et al. (2017), siguiendo a De Alba (2007), plantean que la enseñanza de la EA debe proponer situaciones para el abordaje de problemáticas ambientales atendiendo a su complejidad, desde miradas sociales y políticas de la realidad que inviten a participar de los conflictos ambientales y comprometerse como educador/a. Parten de reconocer a la EA como un "campo emergente conformado por una multiplicidad de actores sociales, enfoques y debates político-pedagógicos que suponen una mirada específica acerca de la cuestión ambiental y la educación" (Canciani et al., 2017, p. 23).

Este enfoque, la Pedagogía del Conflicto Ambiental, pretende integrar la dimensión ambiental en la enseñanza a partir de una mirada crítica y conflictiva de la realidad que, desde la ecología política y el PAL, elabora herramientas pedagógicas para comprender y abordar la compleja trama social en la que se desarrollan los conflictos ecoterritoriales en nuestra región.

Pero, ¿cómo promover instancias de formación docente en torno a la mencionada Pedagogía del Conflicto Ambiental? Desde una perspectiva de EA comunitaria, las acciones educativas se caracterizan como procesos participativos, flexibles y dinámicos que atienden a las demandas locales y problematizan las realidades sociales y lo cotidiano. Estas perspectivas crean redes fuera del ámbito escolar, reconociendo las relaciones colaborativas como una pieza clave en las actividades escolares y extraescolares (Bustos, 2011). Se abren así posibilidades para trabajar con organizaciones sociales del territorio (Castro et al., 2013) en una educación dialógica centrada en las necesidades de las personas y de la sociedad (Carneiro, 2012). En este sentido, desde una perspectiva comunitaria, la formación docente es parte de la relación sistémica entre la escuela y la comunidad.

Para producir estas interacciones dialógicas entre organizaciones sociales y escuelas, hoy en día -y más aún luego de la pandemia que estamos atravesando-, es valiosa la posibilidad de utilizar tecnologías que permitan ampliar los vínculos comunitarios y combinar lo presencial con lo virtual. En algunos casos, los movimientos sociales, paralelamente, utilizan estas nuevas 
herramientas en sentidos contrahegemónicos o de construcciones políticas alternativas (Gendler et al., 2018). Esto genera un gran desafío pedagógico para la educación popular latinoamericana, ya que, como refiere Mejía (2013), incorporar estas tecnologías "desde una mirada crítica emancipadora va más allá de una perspectiva modernizadora por vía del uso y la integración, implica el reconocimiento y la construcción de propuestas transformadoras" (p. 220). Desafíos similares se presentan en las escuelas donde, desde la autonomía docente, sería deseable pasar de la inclusión efectiva - cumplir con un mandato externo- a la inclusión genuina de las TIC planificar con base en un intencionalidad didáctica real por parte de la/el docente- (Maggio, 2012).

\section{Historizando el territorio en disputa}

Desde el punto de vista ecológico, el Delta del río Paraná es un macrosistema de humedales, fuente de valiosos bienes comunes, pero también frágil ante las modificaciones humanas a su hidrología. Allí, además de generarse naturalmente suelo fértil, pesca, medicinas, fibras naturales y otros bienes comunes de la naturaleza, ocurren una variedad de procesos ecológicos beneficiosos para la humanidad (Malvárez, 1999; Kalesnik, 2001).

En sus estudios de historia ambiental de la región, Galafassi (2005) propone la existencia de cuatro períodos bien diferenciados. El primero se remonta a las etnias-naciones chaná y guaraní y finaliza en el siglo XVI con el genocidio perpetrado por los colonizadores europeos. Entre fines del siglo XVI y fines del XIX, la región se mantuvo con una escasa población que, además de vivir de la fauna local, produjo una intensa deforestación del monte blanco, ${ }^{i}$ el bosque nativo originario del Bajo Delta. Entre fines del siglo XIX y mediados del XX, se produjo una gran inmigración de colonos europeos y la región se transformó en una comunidad agrícola -el Bajo Delta llegó a contar con 40.000 personas- que proporcionaba alimentos, madera y otros bienes naturales a Buenos Aires. Entre mediados y fines del siglo XX, luego de una histórica inundación en 1959, y el aumento de la competencia con nuevos centros de producción fruti-hortícola, se produjo una crisis económica y un éxodo masivo de población (en 1970, se contabilizaban unas 3000 personas) (Galafassi, 2005).

Desde fines del siglo XX, podríamos hablar de un nuevo período histórico en términos de cambios en los flujos migratorios, en el cual se produjo una reinvención y disputa por el "paraíso deltaico" (Astelarra, 2016).

Durante estas primeras décadas del siglo XXI, las secciones de islas más cercanas a la megalópoli Buenos Aires han crecido poblacionalmente. Así, la región ha comenzado a enfrentar la amenaza de desarrolladores inmobiliarios (de capitales nacionales y en algunos casos también transnacionales) que especulan con el valor de la tierra y proyectan la creación de mega urbanizaciones de lujo que reemplacen a la población actual. Esta última, portadora de modos de vida isleña, heredera del campesinado originado en los anteriores períodos históricos.

En 2008, se inició el primer conflicto ambiental por urbanizaciones cerradas en el Delta del Paraná, que requirió la intervención de la justicia penal. El proyecto denominado "Colony Park" 
avanzó ilegalmente sobre casi 400 ha de humedales, dirigiendo acciones de intimidación y destrucción de viviendas sobre las familias junqueras y pescadoras residentes en el arroyo Anguilas. En ese año, la población local manifestó su voluntad de resistencia y organización como respuesta a la ola de violentos desalojos llevados a cabo por empresarios en connivencia con agentes estatales y de fuerzas de seguridad. Así, se creó la Cooperativa Isla Esperanza, una organización socioterritorial que se vinculó con el Movimiento Nacional Campesino Indígena (MNCI) (Cooperativa Isla Esperanza, 2012; Astelarra, 2013).

Esta disputa territorial captó la atención mediática ${ }^{2}$ y se fue erigiendo como uno de los conflictos territoriales y ambientales emblemáticos que involucran humedales y negocio inmobiliario en Argentina. En 2012, en un marco de apreciable presión social sobre la dirigencia política local, la justicia federal exhortó a los especuladores inmobiliarios a remediar la totalidad del daño ambiental producido. Sin embargo, el proceso legal iniciado por la Cooperativa Isla Esperanza continúa sin resolución final y la lucha social de las familias junqueras desplazadas y las organizaciones ambientalistas que les apoyan ya cuenta con 13 años de resistencia al desalojo e intento de "colonyzación" del territorio.

A fines de 2016 , la cooperativa y representantes de familias afectadas, a raíz de nuevos episodios de violencia, convocaron a grupos de investigación de diferentes universidades públicas y organizaciones ambientales locales para la conformación de un grupo de apoyo que tomó el nombre de Observatorio de Humedales Delta (OHD). ${ }^{3}$ El Observatorio se conformó como un colectivo de organizaciones y grupos académicos (entre los que se encuentra nuestro grupo de investigación) que integró entonces a: población isleña afectada y organizada en la cooperativa, ambientalistas y activistas locales y personas de la academia de distintas disciplinas, que participan y se involucran en la compleja dinámica de este conflicto ecoterritorial. A partir de 2017, la Cooperativa Isla Esperanza declaró el territorio en conflicto como Reserva Comunitaria Isleña, dando cuenta de una nueva etapa del proceso de reapropiación social del territorio.

\section{Acerca de lo metodológico}

Este trabajo constituye un primer análisis de una experiencia de formación docente comunitaria puesta en marcha conjuntamente con un movimiento socioterritorial, en torno a un conflicto territorial y ambiental complejo y multidimensional. De acuerdo con Messina Raimondi (2008), una posibilidad de investigación sobre la formación docente es la sistematización de experiencias, "la sistematización como un camino para crear saber pedagógico desde la experiencia, la deconstrucción de la experiencia, la producción de un saber colectivo, la crítica radical; la deconstrucción como un proceso de desmontar la realidad" (Messina Raimondi, 2008, p. 11). Según Torres Carrillo (2015), la sistematización de experiencias tiene una forma propia de encontrarse con la práctica. Permite el lenguaje polifónico a través del reconocimiento de las diversas posiciones que expresan los y las participantes y las objetivaciones que adquieren las prácticas. Estas características permiten una particular forma de ingreso de los saberes populares a la investigación. La polifonía no es solo la manera de evidenciar la diversidad y la singularidad, 
es la manera por la cual se reconoce lo valioso de la práctica y los saberes que están presentes en ella. Torres Carrillo y Barragán Cordero (2017) consideran la sistematización de experiencias como una metodología participativa de investigación sobre prácticas significativas de transformación social o educativa que, a partir de su reconstrucción narrativa e interpretación crítica de lógicas y sentidos que las constituyen, busca potenciarlas y rescatar-producir saberes que aportan a las resistencias y re-existencias frente a los modelos hegemónicos. Por ello, decidimos encauzar, desde este marco metodológico, nuestro primer análisis del dispositivo de formación docente comunitaria construido colectivamente. Este marco investigativo propicia el diálogo y el reencuentro entre las personas participantes de una práctica compartida, activando y problematizando los sentidos comunes.

Para reconstruir y analizar la experiencia formativa, la base empírica se sustentó en: registros de videos y audios grabados de instancias de diálogo e intercambio durante los encuentros, notas de campo y otras producciones narrativas elaboradas durante el proceso de sistematización.

\section{Diseño del dispositivo de formación docente: el paso a paso}

Como explicitamos anteriormente, nuestro grupo de investigación (CDC) integra el Observatorio de Humedales Delta (OHD). Desde el GDC, integramos la Comisión de Educación del OHD (CEOHD), conformada por alrededor de 12 participantes, entre habitantes del territorio (isleños/as), ambientalistas y académicos/as provenientes de diversas universidades públicas ubicadas en territorios cercanos, ${ }^{4}$ quienes coincidimos en trabajar desde una perspectiva políticopedagógica enmarcada en la Educación Popular de perspectiva freireana. El proceso de formación docente comunitaria fue planteado inicialmente desde la CEOHD a partir de una instancia de organización comunitaria de reciente conformación: el "Foro de Organizaciones Sociales Isleñas". 5 Allí, durante 2018, distintas organizaciones dialogaron acerca de la necesidad de articular las luchas ambientales y territoriales con las escuelas. En el marco de esta iniciativa, el OHD tejió vínculo con una de ellas, ya que varios/as docentes, madres y padres se encontraban participando del foro. Se trata de una "escuela de islas", pública, de gestión estatal, nivel secundario y modalidad rural (E.E.S. No 12, río Sarmiento, Tigre) ubicada en las cercanías del arroyo Anguilas y el conflicto con la empresa Colony Park.

Dada la urgente necesidad de infraestructura - por compartir sus instalaciones con una escuela de nivel inicial, otra de nivel primario y otra de personas adultas-, parte de la comunidad educativa de esta escuela, desde 2017, conformó un grupo promotor denominado "Secundaria Isleña Sustentable" (SIS) para movilizar la construcción de un nuevo edificio. El grupo SIS plantea basar el proyecto edilicio en criterios de sustentabilidad ambiental, combinado con un nuevo proyecto institucional que articule lo ambiental-territorial con un trabajo sobre las identidades isleñas, la vida comunitaria y los proyectos de vida para las y los jóvenes en el territorio.

A partir del vínculo CEOHD-SIS, se acordó realizar la propuesta de formación que aquí sistematizamos. Integrantes del GDC (entre ellos/as, los/as autores/as de este trabajo), que 
participamos de la comisión de educación junto a otros/as integrantes del OHD, articulamos acciones con el grupo SIS para crear un dispositivo de formación en educación ambiental, orientado a docentes y a la comunidad en general. Este dispositivo didáctico de educación popular se constituyó como un escenario de posibilidad para la emergencia del diálogo de saberes entre la comunidad (cooperativa, isleños/as y vecinos/as de poblaciones urbanas cercanas), Escuela (docentes, familias) y universidad (investigadores/as). Inicialmente, nos basamos en elementos de la Pedagogía del Conflicto Ambiental (Canciani y Telias, 2013; Canciani et al., 2017), teniendo presente que las planificaciones son herramientas flexibles y que la formación ambiental no se restringe al campo pedagógico, sino que requiere integrar, complejizar y articular lo ambiental como dimensión constitutiva de lo social. El dispositivo de formación consistió en un curso, conformado por cuatro encuentros presenciales de cuatro horas de duración cada uno, planificados como talleres de educación popular (Cano, 2012).

El primero de los talleres realizados fue fruto de un proceso previo de elaboración, implementación, análisis y reelaboraciones sucesivas en diferentes contextos socioterritoriales y ambientales de nuestro país. Se implementó una versión inicial con un grupo de docentes de Esquel (Chubut) en diciembre de 2017, contexto atravesado por conflictos ambientales ligados a la megaminería y la industria petrolera. Luego, se efectuó una reelaboración del mismo y fue puesto en práctica con dos grupos de docentes de la ciudad de La Plata (Buenos Aires) en agosto de 2018, donde se trabajó sobre los conflictos ecoterritoriales locales asociados a inundaciones, industria petrolera, agronegocio, especulación inmobiliaria, entre otros (Guerrero Tamayo et al., 2019). Con estas experiencias en haber, en septiembre de 2018, se llevó adelante una nueva reelaboración del taller, que se constituyó en el primer encuentro del dispositivo que analizamos. Se acordó planificar los siguientes talleres a partir de los emergentes de la implementación de este taller inicial. Así, la planificación de los siguientes tres encuentros se fue realizando durante el proceso mismo. Si bien esta fue llevada a cabo principalmente por integrantes del CDC y la CEOHD, fue abierta hacia los y las integrantes de la SIS. Las grandes distancias que separan a las universidades y grupos académicos intervinientes de la ubicación geográfica del territorio en conflicto fueron subsanadas utilizando herramientas TIC colaborativas que, de esta manera, se volvieron estratégicas para la articulación entre organizaciones y el desarrollo del dispositivo didáctico coconstruido. Esta forma de trabajo abierta y flexible requirió de una intensa dedicación a la planificación y socialización interna de las propuestas diseñadas y procesos de reflexión crítica sobre la práctica llevados a cabo en los escasos días disponibles entre los talleres realizados.

Durante este proceso, se originaron diversos espacios participativos (reuniones internas en los diferentes espacios colectivos del CDC, el OHD, la SIS y reuniones plenarias para la planificación colaborativa del trayecto formativo). Esto permitió contar con una planificación general consensuada y, luego, los subgrupos de trabajo existentes (CDC, CEOHD y SIS) fueron activándose con cierta autonomía de acuerdo con las necesidades, prioridades y temporalidades que demandó el proceso en términos de planificación, organización, comunicación y logística, entre otras tareas que fueron necesarias para llevar a cabo los encuentros. La sistematización de 
la experiencia fue realizada, inicialmente, por el GDC y, luego, fue presentada en instancias de participación en la CEOHD, la SIS y a los/as docentes y pobladores/as de la comunidad que participaron de estos encuentros, realizados entre septiembre y noviembre de 2018.

La propuesta de formación fue difundida presencial y virtualmente como "Taller de formación sobre educación ambiental en el Delta de Buenos Aires", un dispositivo de formación de dieciséis horas de duración distribuidas en cuatro encuentros de cuatro horas, dirigida a docentes y pobladores/as del Delta y localidades cercanas. El primer encuentro estuvo centrado en una actividad de mapeo colectivo; el segundo profundizó en conflictos ambientales surgidos del mapeo a distintas escalas; el tercero constituyó una salida de campo al territorio en conflicto; y, el cuarto, un balance del proceso y apertura a elaborar proyectos desde pedagogías del conflicto ambiental.

Una forma de describir sintéticamente la propuesta de formación realizada se resume a continuación (Figura 1):

\section{Figura 1}

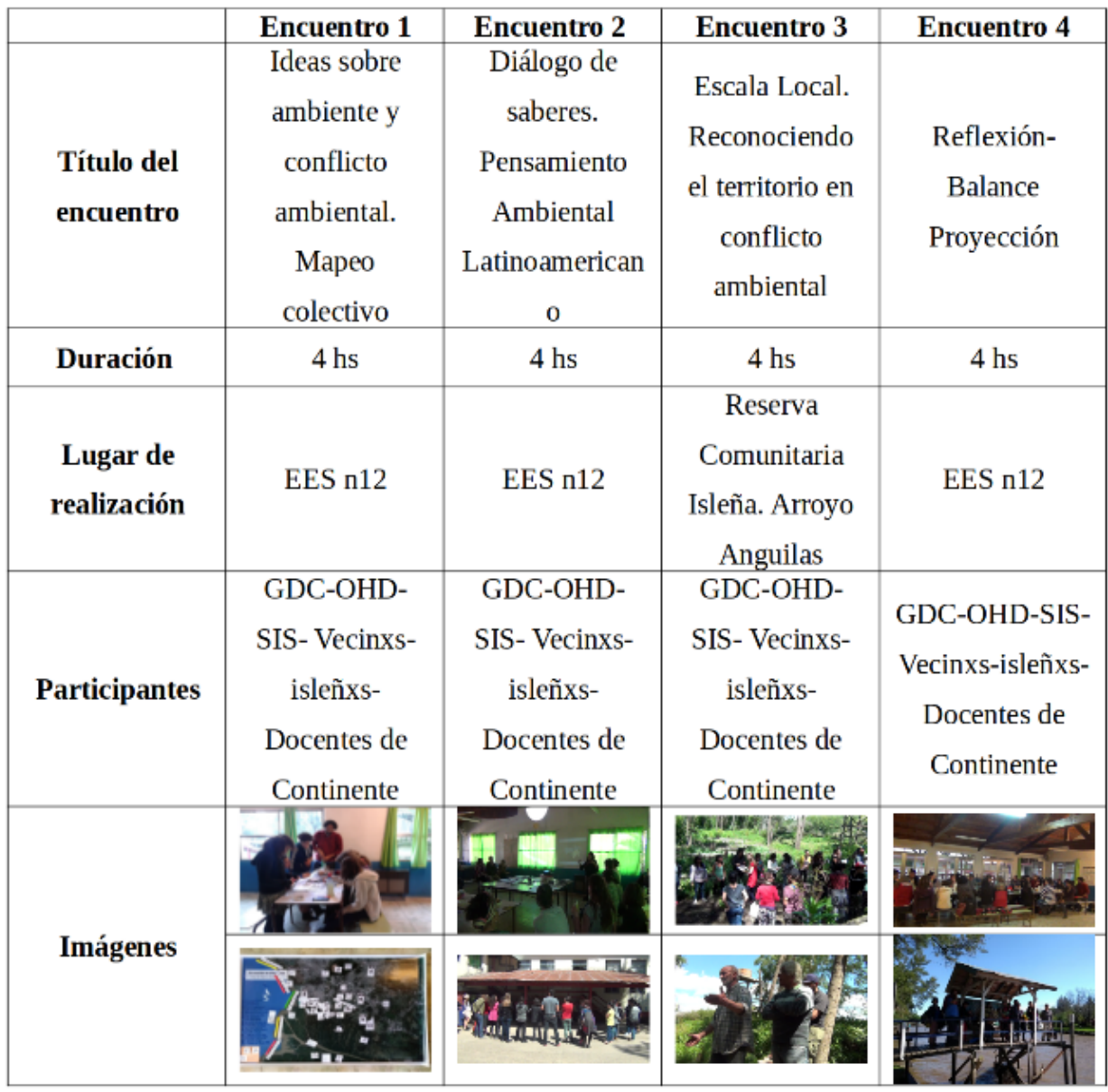




\section{La implementación del dispositivo: vínculos con una Pedagogía del Conflicto Ambiental}

Una de las bases pedagógicas para la elaboración de la propuesta didáctica implementada, como ya dijimos, fue la Pedagogía del Conflicto Ambiental elaborada por Canciani et al. (2017). Los/as autores/as plantean el abordaje de los conflictos ambientales en contextos escolares y proponen lineamientos para la elaboración de propuestas didácticas. Entendemos que esta primera sistematización del proceso formativo implementado se entreteje de diversas formas con esta propuesta teórica de Pedagogía del Conflicto Ambiental. A continuación, recuperamos una enumeración de lineamientos elaborados por los/as autores/as de esta para poner cada uno de ellos en diálogo con la experiencia que implementamos en territorio isleño

- Indagar los diferentes sentidos, percepciones, valoraciones o intereses de los y las participantes sobre el conflicto ambiental a desarrollar. En el primer taller, indagamos acerca de los saberes, ideas y significados de los y las participantes en torno a las ideas de ambiente y conflicto ambiental. "Las 3R", "tomar conciencia", "el reciclado" y "las campañas de ahorro de energía" fueron algunos de los temas que abrieron debates críticos acerca de una concepción ambiental en clave individualista. Los casos de mayor contradicción etico-política en cuanto al sentido de la educación ambiental se discutieron en torno a la participación en actividades implementadas por empresas y organismos estatales y que llevaron a la destrucción del ambiente y los territorios. Si bien la valoración de las prácticas individuales para el cuidado del ambiente fue lo preponderante, algunas voces pusieron el énfasis en la necesidad del abordaje colectivo de los conflictos ambientales.

- Historizar los conflictos ambientales. Es necesario evidenciar que los conflictos ambientales son resultado de procesos históricos, políticos y económicos que pueden ser desentramados y no son procesos ecológicos naturales. Como toda construcción social, es modificable y brinda la oportunidad de pensar y prefigurar otras sociedades. En este sentido, resultó fundamental, desde el punto de vista didáctico en el proceso de mapeo colectivo, rescatar y valorizar el relato de los y las participantes, activando la reconstrucción histórica de la memoria, la memoria popular y la experiencia vivida desde una mirada ambiental. En la escala local de mapeo, se produjo una riqueza mayor de relatos personales, en el reconocimiento de las propias historias de vida permeadas por diversos conflictos ambientales y territoriales. En las escalas de mayor alcance geográfico del mapeo, la historización tuvo un anclaje más ligado a fuentes de conocimiento indirecto (medios de comunicación, conocimiento escolar, etc.).

- Territorializar los conflictos ambientales, ubicarlos en el espacio socioterritorial en que se producen y desarrollan. En torno a este lineamiento, el mapeo colectivo realizado en el primer encuentro incluyó las escalas local, regional y nacional. Para fomentar el intercambio entre grupos, la decisión didáctica fue abordar los mapas de forma interconectada: las y los participantes, organizadas/os en pequeños grupos, intervinieron inicialmente un mapa de una de las escalas $y$, en un segundo momento, trabajaron sobre 
otro de los mapas ya intervenido por otro grupo y, por ende, trabajaron en otra escala de conflictos. Otra decisión didáctica involucró distribuir equitativamente, en los grupos, la pluralidad de voces presentes en la CEOHD, el grupo SIS, el equipo docente y personas de la comunidad que asistieron a los talleres. Estas dinámicas generaron instancias de intercambio que permitieron visualizar los territorios y conflictos desde una diversidad de miradas y, entendemos, proporcionó condiciones para el diálogo de saberes entre las y los participantes.

- Identificar los distintos actores sociales que participan, forman parte, están involucrados o afectados por los conflictos ambientales, dando cuenta de las relaciones, negociaciones, cooperaciones y disputas que existen entre ellos. Las y los participantes, en el mapeo, identificaron conflictos ecoterritoriales locales y regionales, actores y relaciones de poder entre quienes intervienen en los conflictos. En todos los grupos, hubo referencias al accionar de las fuerzas represivas y procesos de militarización en torno a los conflictos ambientales. Particularmente, en la escala local, las y los participantes incluyeron el conflicto con Colony Park e identificaron, por ejemplo, a la Prefectura Naval Argentina que, en los 12 años de conflicto, periódicamente, ha realizado acciones de intimidación de forma sistemática hacia la población que habita el arroyo Anguilas. En la escala regional, se destacó la militarización de la Patagonia y las tierras extranjerizadas mapeadas en la escala latinoamericana. Aparecieron también, en la misma escala, los asesinatos de líderes y lideresas sociales y ambientales, a través de la inclusión del conflicto ambiental que terminó con la vida de Berta Cáceres (lideresa indígena y ambiental de Honduras, que luchaba contra la instalación de un emprendimiento de energía hidroeléctrica de capitales transnacionales). En la Figura 2, se pueden apreciar los íconos y relaciones gráficas establecidas por las y los participantes. El rotulado del mapa con el título: "Las venas abiertas", junto a la lectura de los íconos y las líneas de flujo establecidas, da cuenta del trabajo realizado por los y las participantes en torno al entramado extractivista, los daños producidos sobre los territorios, la consideración de la presencia de fuerzas del estado (y otros grupos paraestatales) y las resistencias. 


\section{Figura 2}

Mapa impreso con el sur apuntando hacia arriba. Escala latinoamericana, intervenido por uno de los grupos

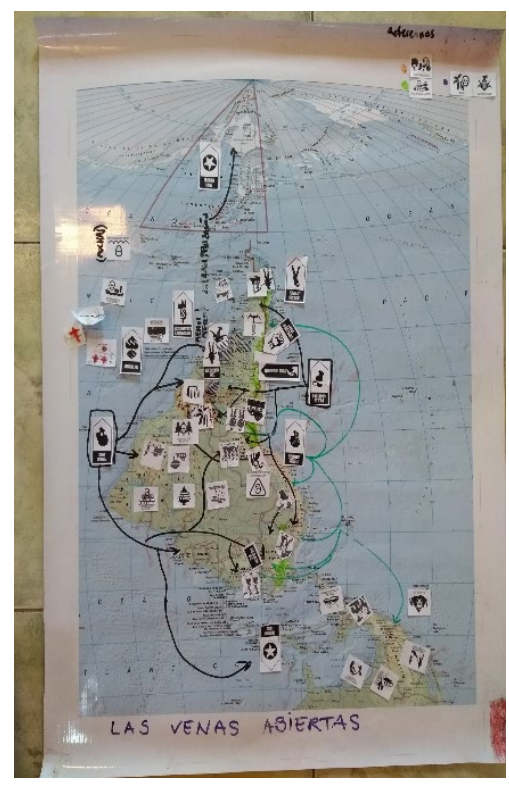

- Incorporar como herramienta de análisis y discusión las normas jurídicas o el marco normativo asociados al conflicto ambiental analizado. El tercer taller se realizó íntegramente en la mencionada Reserva Comunitaria Isleña, autogestionada, en el territorio en conflicto. En uno de los momentos más ricos de los talleres en términos de la pedagogización del conflicto ambiental, una de las integrantes del OHD, isleña e investigadora participante del conflicto ambiental, describió en detalle la dimensión jurídica del conflicto. Relató los pormenores de la causa penal, tramitada en el fuero federal, por daños y estragos a la población, causados por una grave vulneración al ambiente. Dada la influencia de la cuenca del río Paraná, es pasible de ser contemplada como un delito que afecta a toda la población de nuestro país, que ve afectado su derecho constitucional a un ambiente sano. Una de las acusaciones que lleva adelante la población isleña querellante tiene que ver con las lamentables muertes que, periódicamente, se han registrado en los barrios populares afectados por inundaciones extraordinarias en la cuenca del río Luján. En el OHD, existen voces científicas, que se suman al saber popular, que plantean que existe un grado de responsabilidad de las obras de estos megaemprendimientos que rellenan y secan humedales. Durante el desarrollo de la exposición, la investigadora describió el efecto de las megaurbanizaciones sobre la hidrología de los humedales deltaicos que, como resultado, se manifiesta con mayor violencia sobre poblaciones lindantes vulneradas ampliamente en sus derechos. 
- Analizar los conflictos ambientales interdisciplinariamente, considerar las múltiples miradas de conocimientos, saberes y disciplinas. Esta interdisciplinariedad no es la suma de partes fragmentadas, sino un nuevo relato y una reflexión sobre la realidad ambiental analizada. La salida de campo a un territorio en conflicto específico, explicitada en el punto anterior, fue un escenario privilegiado para la interacción y el diálogo entre saberes populares y campesinos y disciplinas académicas. Esta actividad pretendió crear un escenario de posibilidad para la reapropiación social de saberes isleños entre las y los participantes. En ese tercer encuentro, se realizó una caminata hasta un sitio particular en el que es posible, a simple vista, observar los humedales isleños que, con base en las acciones de resistencia, pudieron evitar ser intervenidos por Colony Park, así como el amplio territorio en recuperación dañado por el impacto realizado hace más de una década. La caminata contó con la presencia de integrantes de la Cooperativa Isla Esperanza; biólogos/as, educadores/as y sociólogos/as del OHD, profesores/as, madres y padres de la escuela y vecinos/as. Se realizaron paradas, a modo de postas, en el camino para dar lugar a relatos, preguntas e intercambios transdisciplinares provenientes de las distintas experiencias y saberes. El objetivo pedagógico que persiguió esta actividad fue que la mirada explicitada acerca del conflicto ambiental y territorial contara con una diversidad de saberes entrelazados a través del OHD y la Reserva Comunitaria Isleña.

- Poner en evidencia, a partir de lo relevado, que el conflicto ambiental que se analiza es un conflicto social en tanto está atravesado por una multiplicidad de dimensiones y sujetos quienes, motivados por diferentes intereses, ideologías o creencias, disputan la posesión o el derecho a acceder, administrar o imponer una determinada lógica sobre los bienes comunes. Este lineamiento atravesó los cuatro encuentros. Cada momento de historización y recuperación de la memoria cercana fue acompañado de identificación de actores sociales, intereses y modelos en disputa. Particularmente, en la salida de campo o "entrada al territorio", los y las participantes del curso tuvieron la oportunidad de acceder al relato directo de los y las integrantes de la Cooperativa Isla Esperanza. Ellos/as también describieron la lucha territorial y legal que llevan adelante frente al grupo empresarial que intentó expulsarlos/as completamente de su territorio. Algunos/as pobladores/as han regresado y reconstruído sus características viviendas isleñas, otros/as aún tienen miedo o grandes dificultades económicas y continúan esperando una hipotética sentencia judicial favorable. Otros/as han muerto y continúan la lucha sus familiares.

- Realizar un manejo crítico de la información disponible. Consultar y analizar diversas fuentes de información e interpretar críticamente cómo responden a diferentes intereses sociales y económicos. En nuestra experiencia, abordamos diversidad de fuentes de información para el análisis de conflictos ecoterritoriales en las distintas escalas trabajadas en el taller de mapeo colectivo. Particularmente, durante el segundo encuentro, se trabajó con base en la facilitación de un banco de recursos TIC para la profundización y el estudio de casos. 
- Interpelar y movilizar a los sujetos hacia una reflexión que involucre no solo la necesidad de aportar una "solución" en términos técnicos e individuales, sino más bien la posibilidad de apostar a una construcción social y colectiva para un proyecto más justo y sustentable, que apunte a la transformación de las desigualdades sociales que se encuentran de fondo en las luchas ambientales latinoamericanas. En nuestro caso, particularmente, la "entrada al territorio en conflicto", las interacciones e intercambios con los/as propios/as damnificados/as y su organización cooperativa fueron valoradas durante el balance posterior (realizado por los y las participantes) como una manera de conocer en profundidad un conflicto territorial cercano y visibilizar posibilidades y alternativas. Los y las integrantes de la Cooperativa explicitaron, en varias oportunidades, la importancia de haberse organizado para esa lucha ambiental, evidenciando la relevancia de la acción colectiva y colaborativa para los movimientos socioterritoriales que nutren la reflexión de la ecología política latinoamericana.

- Analizar los procesos de construcción colectiva en términos de mediano y corto plazo, así como incorporar a la propuesta la relación entre educación ambiental y derechos humanos. La decisión didáctica de iniciar una historización del territorio en el primero de los talleres y retomarla durante los subsiguientes para ampliarla y profundizarla, así como la recuperación de la memoria individual y colectiva durante la "entrada al territorio", apuntaron en este sentido. En particular, en este caso, la relación entre lo ambiental y derechos humanos, tal como explicitaron los y las integrantes de la cooperativa durante ese taller, se pone de manifiesto en la misma causa judicial. En ella, una de las líneas de acusación plantea que el Estado ejerció una violencia sistemática para expulsar y erradicar del territorio en conflicto a la población isleña a través de la complicidad de diferentes organismos estatales y funcionarios públicos, constituyéndose por dicho carácter sistemático en contra de una población discriminada y violentada por ser portadora de rasgos sociales y culturales específicos en un crimen de lesa humanidad.

\section{Aportes a las pedagogías del conflicto ambiental}

Además de los vínculos con la Pedagogía del Conflicto Ambiental que tomamos como marco teórico y didáctico de Educación Ambiental, sistematizamos algunos aportes que son particulares de esta experiencia y no forman parte de los lineamientos explicitados en la propuesta de Canciani et al. (2017). Esto nos permite pensar en contribuir a la construcción de pedagogías del conflicto ambiental en un sentido ampliado y diverso.

Como adelantamos anteriormente, desde el inicio de los talleres, tomamos decisiones didácticas que fueron integrando el trabajo con las TIC desde una perspectiva de inclusión genuina (Maggio, 2012). Una de las integraciones didácticas de recursos TIC fue el uso de cortos audiovisuales ambientales que circulan en las redes sociales. Durante el primer taller, se seleccionaron y visionaron dos cortos que presentaban miradas antagónicas respecto al ambiente y los conflictos ambientales, con la intencionalidad de promover algunas preguntas y debates 
iniciales en torno a la idea de conflicto ambiental, así como propiciar miradas en clave de género que se entretejen con la cuestión ambiental.

En términos de diseño y organización de las actividades propuestas durante el curso, fue necesario realizar búsquedas en sistemas de información geográfica (SIC) que son de acceso público en la web y seleccionar mapas de alta calidad para ser impresos. Los mapas digitales en formato físico se utilizaron como recurso didáctico para las dinámicas grupales de mapeo colectivo, en las que se promovió la intervención libre y creativa con variados elementos para escribir, dibujar o generar collages sobre los mapas, que luego pudieron fotografiarse y limpiarse para su reutilización. Para todo ello, fue necesario aprender el manejo de software específico para descargar e intervenir los mapas digitalmente antes de disponibilizarlos, ya que una de las intencionalidades fue poner en cuestión la propia construcción de las escalas y proyecciones desde una perspectiva de cartografía social crítica.

Otro imprescindible uso de las TIC estuvo reflejado en la coordinación colectiva de la "entrada al territorio". Se acordó con las y los participantes crear un grupo de mensajería instantánea a través de los teléfonos móviles para acordar y comunicarse de manera ágil y colectiva en torno a los detalles logísticos del traslado, contingencias, cambios por cuestiones climáticas, etc. Luego, se amplió la finalidad de dicho grupo virtual, y fue usado como herramienta de comunicación para los siguientes avances en el proceso de formación. Asimismo, se amplió al uso de un Padlet, un recurso TIC en línea, diseñado como espacio de colaboración, a través del cual se compartieron textos, videos, mapas y otros recursos sobre conflictos ambientales y Educación Ambiental. También este canal de comunicación fue clave para la organización de un reencuentro al año siguiente (2019) en el que se realizó una devolución (comunicación-acción a todo el grupo participante) de la sistematización realizada. De igual forma, el uso de herramientas TIC para la planificación colaborativa en y entre los grupos (CDC, CEOHD, SIS), a lo largo de todo el proceso, además de sostener los vínculos grupales, promovieron la escritura colaborativa (Dillenbourg, 1999) más allá del contexto universitario, afianzando una interacción dentro y fuera del territorio.

Una segunda perspectiva no considerada explícitamente por los lineamientos de Canciani et al. (2017) y que, aún incluida incipientemente en la planificación, cobró relevancia a partir del análisis de lo acaecido en los encuentros fue la perspectiva de género. En el primer encuentro, luego de las presentaciones de los y las participantes y explicitación de la intencionalidad del dispositivo de formación, proyectamos (como dijimos más arriba) dos videos que planteaban concepciones antagónicas respecto al ambiente y la participación individual y colectiva en su cuidado o destrucción. Al proyectar los videos, una de las cuestiones que se propuso analizar fue la perspectiva de género subyacente a cada corto ambiental. En el trabajo en pequeños grupos, surgieron ejemplos locales que pusieron en evidencia formas en las que el patriarcado ejerce violencia sobre los cuerpos de las mujeres y cómo se relaciona con la cuestión ambiental. Surgió el análisis de situaciones vividas por las participantes y otras mujeres del territorio, al ser maltratadas recurrentemente por "los lancheros" (forma de nombrar a los precarizados 
trabajadores que conducen y tripulan las lanchas que funcionan como transporte público de pasajeros). Estas situaciones y lógicas se vincularon con el rol que juegan los trabajadores varones isleños en relación a los daños ambientales. Dichos varones isleños son los que han conducido dragas y palas mecánicas (a cargo de Colony Park) para destruir un inmenso territorio de humedales prácticamente sin considerar a sus propios vecinos/as que habitaban el territorio, sus viviendas y vivencias. Esta situación fue orquestada por un poderoso empresario, varón también, denunciado por violentas prácticas mafiosas en reiteradas oportunidades. En estos debates intragrupales, se discutió también acerca de los estereotipos de género que aparecen ligados a los roles de explotación-saqueo/cuidado del ambiente-cuidado de la naturaleza. Asimismo, durante el mapeo colectivo, en las escalas local y nacional, algunas mujeres participantes promovieron debates sobre los derechos al territorio, la autonomía, la soberanía alimentaria, el reconocimiento de los derechos de los diversos grupos de mujeres (isleñas, indígenas, rurales, urbanas, negras), los derechos sexuales y reproductivos, el autocuidado y el autoconocimiento.

\section{Reflexiones desde la pedagogización de un conflicto ambiental}

La sistematización realizada nos sugiere que la propuesta de Canciani et al. (2017) de Pedagogía del Conflicto Ambiental, sumada a la participación de una organización socioterritorial en la planificación del proceso educativo y la implementación de los encuentros de formación, permitieron configurar un dispositivo didáctico de formación docente en EA que promovió la participación diversa en la reapropiación social del territorio desde el punto de vista pedagógico. Este proceso, que entendemos de pedagogización del conflicto ambiental, tuvo algunas características singulares.

Una característica particular de nuestra experiencia educativa fue que no se originó desde la escuela hacia la comunidad, sino que surgió de la interacción entre organizaciones socioterritoriales. Estas constituyeron el punto de partida para diseñar y concretar el proceso de formación docente en ejercicio y la elaboración de la propuesta didáctica que sería implementada. Los y las integrantes de la Cooperativa Isla Esperanza están convencidos/as, y propugnan, que niños, niñas y jóvenes se formen para la defensa del territorio y entienden que la escuela es un lugar central en esta tarea. Ellos/as fueron quienes, desde la constitución del OHD, promovieron e insistieron en que se realizaran procesos de articulación con escuelas. Su compromiso permitió la "entrada al territorio" y los relatos en primera persona, indispensables para compartir vivencias.

Otras dos características fueron originales en nuestro contexto: por un lado, que el proceso de formación docente fuera coordinado por personas que trabajamos en el ámbito académico en los campos de la educación y la educación ambiental junto a vecinos/as (que participan del OHD). Por otro, que incluyera madres, padres y vecinos/as de la comunidad como participantes junto al equipo docente. Ambas características promovieron la ampliación de los debates, el compartir de saberes, vivires y sentires más allá del ámbito escolar y la identificación de actores y miradas posibles de coordinarse o articularse para la defensa del territorio. 
El análisis del dispositivo en su conjunto, enfocando la mirada en la explicitación e intercambio de saberes portados por los/as diferentes participantes como vía para la construcción de un saber ambienta/ (Leff, 1998) tuvo diferentes momentos e instancias propiciadas por la conformación diversa del grupo. De estas instancias, la más rica en cuanto a cantidad y diversidad de voces participantes fue la salida de campo o, como preferimos llamarla, "entrada al territorio". En esta actividad, resultó crucial la presencia de integrantes de la Cooperativa Isla Esperanza, que residen desde hace varias generaciones en el territorio y se han constituido en la organización socioterritorial que evitó el avance violento de los especuladores inmobiliarios. Esta participación, por un lado, abrió la posibilidad de re-construir y recuperar saberes acerca del daño ambiental y sus posibilidades de reparación desde la perspectiva de quienes habitan el territorio. Por otro, constituyó la oportunidad de evidenciar la valoración epistémica de los saberes isleños en el espacio formativo, al promover su abordaje junto con el conocimiento científico y jurídico. Docentes y pobladores/as isleños/as de diversos sectores sociales participaron de un diálogo en el que se escucharon e intercambiaron voces provenientes de fuentes diferenciadas. Se intentó, de esta manera, aportar a la desnaturalización de la jerarquía social que posee el conocimiento científico por sobre el saber popular. Sin embargo, es preciso recordar que, como advierten Merçon et al. (2014), en estos diálogos de saberes y vivires, es difícil desmontar completamente las relaciones de poder que se encuentran firmemente cimentadas en nuestra sociedad. Sabemos que no se trata de un proceso instantáneo ni acabado, pero esperamos haber contribuido a un nuevo giro de reapropiación social del territorio en conflicto a través de la acción educativa. Desde la perspectiva de Leff (2014), estos procesos de diálogo de saberes se constituyen en una vía para la construcción de saber ambiental. Según este autor, el diálogo de saberes parte del intercambio de miradas y opiniones sobre las cuestiones que aquejan al mundo y en las cuales están comprometidos diferentes intereses y son confrontados puntos de vista diversos; propicia un intercambio intercultural; y promueve la fusión entre diferentes disciplinas con el propósito de construir esquemas inter o transdisciplinarios de conocimiento. Pero además, y centralmente, funda un sentido categorial que refiere al encuentro entre diferentes seres culturales, a la diversidad de modos de comprensión del mundo y de modos de ser en el mundo.

Dentro de las distintas formas en las que los y las participantes se posicionaron frente a la crisis ambiental planetaria y la conflictividad, recuperadas a través del mapeo, le otorgamos una relevancia significativa a la tensión que identificamos entre enfoques individualistas y colectivos que coexisten en diferentes tendencias de educación ambiental. En la escala local, se pasó de mapear prioritariamente "problemáticas ambientales", como el tratamiento de la basura y la contaminación, a profundizar en los conflictos por la tenencia de la tierra, que implican la afectación directa a los modos de vida anidados en el territorio. Así, luego de discusiones, reflexiones y tensiones colectivas, se dio apertura al abordaje de las luchas por el derecho a ser (isleños/as), por el derecho al territorio (un espacio para ser), el derecho a la autonomía, a construir un futuro desde las diferentes visiones culturales, formas tradicionales de producción (junco) y organización social (los/as junqueros/as). En la expresión de estas tensiones, 
nuevamente, identificamos la posibilidad de emergencia del diálogo de saberes descrito por Leff $(2014,2018)$ y de reconocimiento de las tramas sociopolíticas que Machado Aráoz (2013) describe en torno a los movimientos ecoterritoriales de latinoamérica.

Los debates intragrupales identificados en el proceso de sistematización, relacionados con las violencias vividas por las mujeres en las actividades específicas y cotidianas del territorio, se vincularían con reflexiones y elaboraciones producidas desde los feminismos populares latinoamericanos (Svampa, 2015).

Si bien el disparador para el debate durante el taller estuvo constituido por situaciones externas (los cortos animados), la reflexión se situó en contexto, a partir de las propias experiencias vividas en los cuerpos de las mujeres y en el territorio. Aún cuando no fue profundizada durante los talleres, la emergencia de reflexiones incipientes acerca del patriarcado como sistema de opresión no solo sobre las mujeres, sino también sobre la naturaleza, así como de debates sobre derechos al territorio, autonomía, derechos sexuales y reproductivos, autocuidado y autoconocimiento, constituirían "pistas" (Guelman y Palumbo, 2018) que orientan en el reconocimiento de la categoría "cuerpo-territorio" (Cabnal, 2016) como una posible puerta de entrada a la racionalidad ambiental propuesta por Leff $(2002,2014,2018)$.

El reconocimiento político-epistémico de vínculos, experiencias vividas y relaciones situadas nos aparta de la racionalidad cartesiana y nos acerca a los feminismos populares (Dumrauf et al., 2019). La categoría "cuerpo-territorio" ayuda a analizar cómo las violencias dejan marcas en los cuerpos (Lopes Louro, 2004), pero, además, se conecta con los daños y conflictos en los territorios (Cabnal, 2016).

Asimismo, el dispositivo de formación docente evidenció la potencia de incorporar las TIC enfocadas desde los modos de apropiación de los movimientos socioterritoriales. Esto involucra no solo la mencionada "inclusión genuina" (Maggio, 2012), sino también la "construcción de agendas críticas" para el uso de TIC desde la mirada de la educación popular (Mejía, 2013). El acceso a través de internet a material audiovisual de contenido crítico producido por artistas y comunicadores/as visuales en torno a un conflicto ambiental por el agua (The Animation Workshop/Colectivo de Animadores Bolivianos, 2010) colaboró en activar debates con el aporte de miradas provenientes de la ecología política de los conflictos ambientales. También, estos materiales posibilitaron una apertura de diálogo acerca de las cuestiones de género que atraviesan lo ambiental. Igualmente, los recursos digitales y visuales: videos, pictogramación, iconografías, dispositivos gráficos y cartográficos (Iconoclasistas, 2013), cobraron significado como formas ampliadas de comprensión, reflexión y visibilización de aspectos prioritarios para la EA latinoamericana. Estos recursos, al ser utilizados en dinámicas grupales que combinaron el plenario con el trabajo en pequeños grupos, permitieron construir enunciados que emergieron del diálogo de saberes y vivires de los y las participantes (Leff, 2014; Merçon et al., 2014). De esta manera, nos estaríamos alejando de un uso de las TIC tecnicista e instrumental. 


\section{A modo de cierre provisorio}

¿Cómo articular organizaciones sociales en lucha con la escuela? ¿Cómo con instancias de formación docente en ejercicio? ¿Qué aportaría, si lo hiciera, este proceso de trabajo conjunto?

A través de una primera sistematización de un proceso de formación docente en ejercicio surgido a instancias de, e implementado con, una organización socioterritorial, entendemos que aportamos a la comprensión de algunas formas en las que estos movimientos pueden contribuir a los marcos pedagógico-didácticos que sustentan diálogos entre escuela y comunidades. Particularmente, rescatamos los aportes a la pedagogización de un conflicto ambiental. Telías et al. (2017) proponen "conflictivizar las problemáticas ambientales", es decir, pasar de considerar el estudio de "problemáticas ambientales" a involucrarse en el estudio y vinculación escolar con "conflictos ambientales". El análisis que presentamos nos sugiere ampliar esa propuesta. La pedagogización de los conflictos ambientales se nutre y complejiza con la participación de los movimientos en lucha ambiental en acciones educativas de formación docente y en la construcción de la educación ambiental que ocurre en las escuelas de los territorios. Este enfoque pedagógico del conflicto ambiental desde el movimiento socioterritorial hacia la escuela propiciaría escenarios de diálogo de saberes que se proponen desde el Pensamiento Ambiental Latinoamericano para la construcción de saber ambiental (Leff, 1998). Articularía también con las reivindicaciones ético-políticas de los movimientos socio-teritoriales. Estas reivindicaciones, y las causas profundas de la injusticia, no son diferentes de las de los demás grupos de oprimidos del mundo que vienen luchando por la emancipación. El ambiente -o el cuerpo/territorio- es uno de los espacios en disputa. 


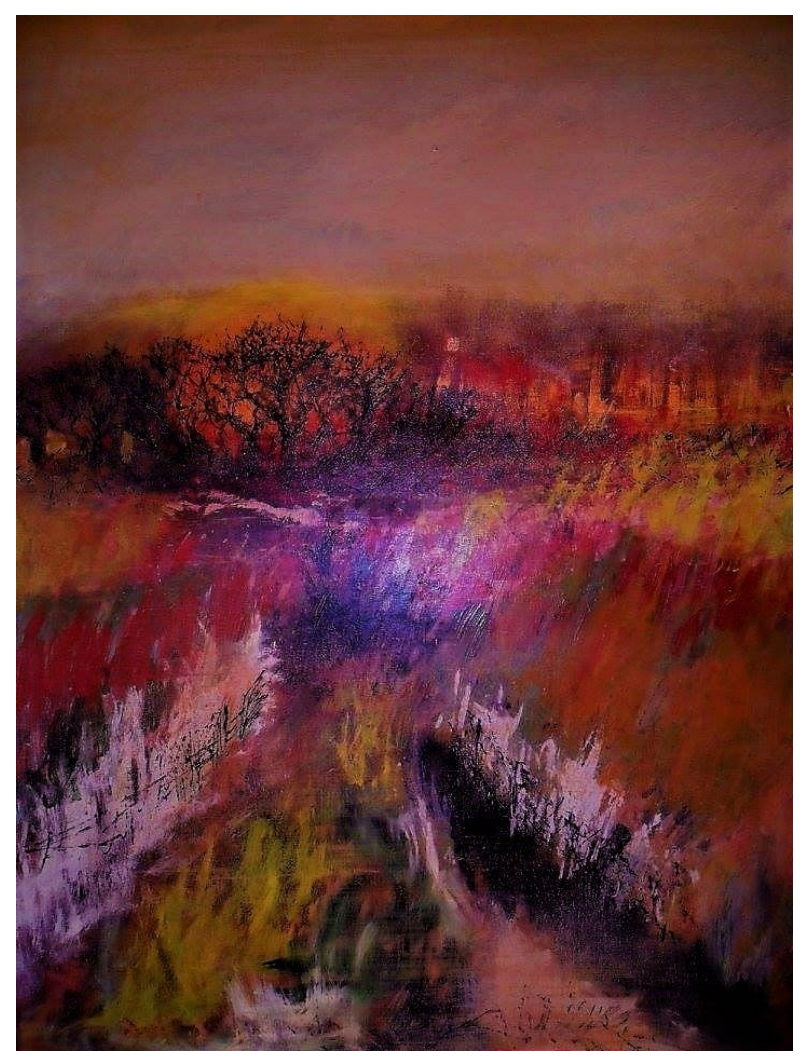

Atardecer en rojo, técnica mixta. Adriana Chavarri

\section{Bibliografía}

Astelarra, S. (2013). "No ColonYzarán". Conflictos por la apropiación social de la naturaleza en el Bajo Delta del Paraná. X Jornadas de Sociología. Facultad de Ciencias Sociales, Universidad de Buenos Aires.

Astelarra, S. (2016). Disputas por la reinvención del "paraíso deltaico": de los lugares de la querencia a llegar a una isla y olvidarse de todo. El caso del conflicto "Colony Park" en la primera sección de islas del Delta del Paraná. En G. Merlinsky (Comp.), Cartografías del conflicto ambiental en Argentina 2. CLACSO.

Bustos. (2011). Escuelas rurales y educación democrática. La oportunidad de la participación comunitaria. REIFOP, 14(2), 105-114.

Cabnal, L. (2016). Feminismos diversos: el feminismo comunitario. ACSUR-Las Segovias.

https://porunavidavivible.files.wordpress.com/2012/09/feminismos-comunitario-lorena-cabnal.pdf Cano, A. (2012). La metodología de taller en los procesos de educación popular. Revista Latinoamericana de Metodología de las Ciencias Sociales, 2, 22-52

Carneiro. (2012). Las TIC y los nuevos paradigmas educativos: la transformación de la escuela en una sociedad que se transforma. En R. Carneiro, J. C. Toscano y T. Díaz (Eds.), Los desafíos de las TIC para el cambio educativo (pp. 15-28). OEI-Fundación Santillana.

Castro Estrada, M. L., Rejas, M. J. R. y Urteaga Urías, E. (2013). Abriendo las aulas: la vinculación entre docencia, investigación y cooperación comunitaria. Revista Electrónica Interuniversitaria de Formación del Profesorado, 16(3), 33-47.

Cooperativa Isla Esperanza/Universidad Nacional de Luján. (2012). Isla Esperanza. Trabajo, naturaleza y resistencia isleña. UNLU. 
Canciani, M. L. y Telías, A. (2013). Aportes teóricos conceptuales para pensar los procesos educativos en escenarios de conflicto ambiental. Revista del IICE, (34), 111-122.

Canciani, M. L., Telías, A. y Sessano, P. (2017). Problemas y desafíos de la educación ambiental. Un abordaje en 12 lecciones. Ediciones Novedades Educativas.

Corbetta, S. (2015). Pensamiento Ambiental Latinoamericano y Educación Ambiental. Voces en el Fénix, 43, 160-167.

De Alba, A. (2007). Investigación en educación ambiental en América Latina y el Caribe. Doce tesis sobre su constitución. En G. González Gaudiano, La educación frente al desafío ambiental global: una visión latinoamericana (pp. 277-287). Plaza y Valdés-Crefal.

Dillenbourg, P. (1999). What do you mean by collaborative learning? En P. Dillenbourg (Ed), Collaborativelearning: Cognitive and Computational Approaches (pp. 1-19). Elsevier.

Dumrauf, A. y Mengascini, A. (2008). Los Movimientos Sociales en América Latina. Pasado, presente y perspectivas. Memorias de las Jornadas de Problemas Latinoamericanos, 1732-1739.

Dumrauf, A., Cordero, S. y Mengascini, A. (2013). De docentes para docentes: experiencias innovadoras en ciencias naturales en la escuela pública. El Colectivo.

Dumrauf, A., Cordero, S. y Mengascini, A. (2016). Experiencias educativas de los movimientos sociales: Contribuciones para la educación científica, ambiental y en salud desde una perspectiva emancipadora. Revista Brasileira de Pesquisa em Educação em Ciências, v. 16 , no. 2, 477-497.

Dumrauf, A. y Cordero, S. (2018). Tramas entre escuela y universidad: formación docente, innovación e investigación colaborativa. EDULP. http://sedici.unlp.edu.ar/handle/10915/69683

Dumrauf, A. y Cordero, S. (2020). Un enfoque participativo para la formación docente continua en la Educación en Ciencias Naturales, Ambiental y en Salud. Revista Eureka sobre Enseñanza y Divulgación de las Ciencias, v. 17, no. 1, 1-15.

Dumrauf, A., Cordero, S., Cucalón Tirado, M. P, Guerrero Tamayo, K. y Garelli, F. (2019). Hacia nuevos territorios epistémicos: aportes desde un camino de construcción pedagógica descolonizadora en Educación en Ciencias Naturales, Ambiental y en Salud. En B. Monteiro, Dutra, D., y Cassiani, S., Decolonialidade na Educação em Ciencias. Livraría da Física.

Escobar, A. (2018). Otro posible es posible: Caminando hacia las transiciones desde Abya Yala/Afro/LatinoAmérica. Ediciones Desde Abajo.

Freire, P. (1978). Pedagogía del oprimido. Siglo XXI.

Freire, P. (2012). Pedagogía de la indignación: cartas pedagógicas en un mundo revuelto. Siglo XXI Editores. Galafassi, G. (2005). La pampeanización del delta, sociología e historia del proceso de transformación productiva, social y ambiental del Bajo Delta del río Paraná. Ediciones Extramuros.

Carelli, F., Cordero, S., Mengascini, A. y Dumrauf, A. (2019). Caminos para la Educación en Salud desde la Educación Popular: experiencias en Bachilleratos Populares de Argentina. Ciência \& Educaçao, vol. 25, no. 1, 5-24.

Gendler, M. A., Méndez, A., Samaniego, F. y Amado, S. (2018). Uso, apropiación, cooptación y creación: pensando nuevas herramientas para el abordaje de la Apropiación Social de Tecnologías. En S. Lago Martínez, A. Álvarez, M. Gendler y A. Méndez, Acerca de la apropiación de tecnologías: teoría, estudios y debates. Ediciones Del Gato Gris.

Guelman, A. y Palumbo, M. M. (2015). Prácticas pedagógicas descolonizadoras en experiencias productivas autogestionadas de movimientos sociales: el principio formativo del trabajo. Revista Interamericana de Educación de Adultos, vol. 37, no. 2, 48-64. 
Guelman, A. y Palumbo, M. M. (Coord.). (2018). Pedagogías descolonizadoras. Formación en el trabajo en los movimientos populares. Editorial El Colectivo-CLACSO.

Guerrero Tamayo, K., Iribarren, L., Dumrauf, A., Ollier, S. y Mengascini, A. (2019). Educación ambiental en la formación docente: aportes para un abordaje multidimensional de los conflictos ambientales. XII Encontro Nacional de Pesquisa em Educação em Ciências. XII ENPEC Universidade Federal do Rio Grande do Norte, Natal, RN. 25 a 28 de junio de 2019.

Iconoclasistas. (2013). Manual de mapeo colectivo. Recursos cartográficos críticos para procesos territoriales de creación colaborativa. Tinta Limón.

Kalesnik, F. (2001). Relación entre la heterogenidad ambiental y los neoecosistemas de albardón (bosques secundarios) en las islas del Bajo Delta del Río Paraná. Tendencias de regeneración y composición futura [Tesis doctoral]. Buenos Aires: Facultad de Ciencias Exactas y Naturales. Universidad de Buenos Aires. Leff, E. (1998). Saber ambiental. Racionalidad, sustentabilidad, complejidad, poder. Siglo XXI Editores. Leff, E. (2014). La Apuesta por la Vida. Imaginación sociológica e imaginarios sociales en los territorios ambientales del sur. Vozes Editora.

Leff, E. (2018). El fuego de la vida: Heidegger ante la cuestión ambiental. Siglo XXI Editores. Lopes Louro, G. (2004). Um corpo estranho: ensaios sobre sexualidade e teoria queer. Autêntica. Loureiro, C. F. B. (2003). Emancipación, complejidad y método histórico dialéctico: repensar las tendencias en educación ambiental. Tópicos en Educación Ambiental, vol. 5, no. 13, 21-30.

Machado Aráoz, H. (2013). Extractivismo y "Consenso Social": Expropiación-consumo y fabricación de subjetividades (capitalistas) en contextos neocoloniales. Revista Cuestiones de Población y Sociedad, Córdoba, vol. 3, no. 3, 29-42.

Maggio, M. (2012). Entre la inclusión digital y la recreación de la enseñanza: el modelo 1 a 1 en Argentina. Campus Virtuales, vol. 1, no. 1, 51-64.

Malvárez, A. I. (1999). El Delta del Río Paraná como mosaico de humedales. En A. I. Malvárez, Topicos Sobre Humedales Subtropicales y Templados de Sudamérica (pp. 5-53). MAB-ORCYT.

Mejía, M. R. (2013). La educación popular con y desde las NTIC. En L. Cendales, M. R. Mejía y J. M. Muñoz (Eds.), Entretejidos de la educación popular en Colombia. CEEAL (pp. 185-222). Ediciones desde abajo.

Mejía, M. R. (2014). La Educación Popular: Una construcción colectiva desde el Sur y desde abajo. Arquivos Analíticos de Políticas Educativas, vol. 22, no. 62, 1-30.

Merçon, J., Camou, A., Nuñez, C. y Escalona, M. (2014). ¿Diálogo de saberes? La investigación acción participativa va más allá de lo que sabemos. Decisio. Saberes para la acción en Educación de Adultos, 38, 2933.

Messina Raimondi, G. (2008). Formación "docente": del control al saber pedagógico. Docencia, 34, 78-86. Puiggrós, A. (2005). De Simón Rodríguez a Paulo Freire: educación para la integración iberoamericana. Convenio Andrés Bello.

Rockwell, E. (2012). Movimientos sociales emergentes y nuevas maneras de educar. Educação \& Sociedade, Campinas, vol. 33, no. 120, 697-713.

Svampa, M. (2015). Feminismos del Sur y ecofeminismos. Nueva Sociedad 256, https://nuso.org/articulo/feminismos-del-sur-y-ecofeminismo/. The animation workshop/Colectivo de Animadores Bolivianos. (Dir.). (2010). La abuela grillo. Center Animation Pedagogic. Disponible en https://www.youtube.com/watch?v=AXz4XPuB_BM F/c: 25/05/2020 Torres Carrillo, A. (2015) Educación Popular y movimientos sociales en América Latina. Biblos. 


\section{Agradecimientos}

A los/as compañeros/as de la Cooperativa Isla Esperanza, el Observatorio de Humedales Delta y el grupo de la Secundaria Isleña Sustentable, quienes sueñan y promueven mundos plurales y justos, y nos invitan e incentivan a construir con ellos/as. A los/as docentes, madres, padres y vecinos/as que nos recibieron generosamente, nos abrieron y nos hicieron partícipes de sus debates y proyectos.

\footnotetext{
' Selva en galería característica del Bajo Delta, caracterizada inicialmente por Burkart (1957) y estudiada posteriormente por Kalesnik (2001) y Sirolli (2018).

${ }^{2}$ Ver Malojillo films. (2010). Junqueros. Cooperativa Isla Esperanza, familias isleñas resisten al desalojo ilegal de Colony Park https://vimeo.com/12249068, también, Vaisman, M. (2013). El Delta, Historias del Río. https://www.youtube.com/watch?v=QyD9clznb4k, Visión 7. (2013). La resistencia de los isleños https://www.youtube.com/watch?v=oD8wrwNs5Ss ${ }^{3}$ https://www.facebook.com/ObservatorioHumedalesDelta/ ${ }^{4}$ Universidad Nacional de La Plata, Universidad Nacional de Luján, Universidad Nacional de San Martín y Universidad de Buenos Aires.

${ }^{5}$ Se trata de encuentros mensuales donde participan centros culturales, radios y centros comunitarias, organizaciones ambientalistas, organizaciones feministas, sindicatos y otras organizaciones sociales del Delta del Paraná.
} 